\title{
PRACTICAL APPROACH TO THE EVALUATION AND MANAGEMENT OF RHEUMATOID ARTHRITIS-INTERSTITIAL LUNG DISEASE BASED ON ITS PROVEN AND HYPOTHETICAL MECHANISMS
}

\author{
Francisco Paulin ${ }^{1 *}$, Alejandra Babini ${ }^{2}$, Marta Mamani $^{3}$, Juan Mercado $^{4}$ and Fabián Caro ${ }^{1}$ \\ ${ }^{1}$ Interstitial Lung Disease Clinic, Hospital María Ferrer, Buenos Aires, Argentina; ${ }^{2}$ Division of Rheumatology, \\ Hospital Italiano, Córdoba, Argentina; ${ }^{3}$ Division of Rheumatology, Hospital Rivadavia, Buenos Aires, Argentina; \\ ${ }^{4}$ Horizon Hospital, University of Pittsburgh Medical Center, PA, USA
}

\begin{abstract}
The prevalence of interstitial lung disease in patients with rheumatoid arthritis varies from 10 to $42 \%$. Rheumatoid arthritis patients with interstitial lung disease have three times the risk of death compared with those without the disease. Prognosis seems to be related to the high-resolution computed tomography pattern. Usual interstitial pneumonia pattern, resembling idiopathic pulmonary fibrosis, carries a worse prognosis. Validated strategies to identify different phenotypes and assess the disease activity in rheumatoid arthritis interstitial lung disease are lacking. However, the utilization of high-resolution computed tomography, composed disease activity scores, and anti-citrullinated peptide antibodies titers can help to guide decisions in clinical practice. Mechanisms involved in lung disease may be different from those implicated in joint involvement. This could explain why in a significant proportion of cases, interstitial lung disease does not improve or even worsens with standard therapies used successfully to treat the joint component (e.g. anti-tumor necrosis factor agents). In this scenario, a group of drugs that targets the adaptive immune response (e.g. rituximab or abatacept) seems to target more specifically the process that takes place in the lungs. Moreover, the recent emergence of anti-fibrotic drugs, which have already proven effective in idiopathic pulmonary fibrosis, may provide an alternative treatment strategy in rheumatoid arthritis-usual interstitial pneumonia. In this review, we propose a practical approach to the evaluation and therapy of rheumatoid arthritis interstitial lung disease. Validation of strategies directed to assess the activity of lung disease and identify the underlying mechanisms are needed. Clinical trials evaluating a therapeutic approach with specific targets based on the disease phenotype are warranted.
\end{abstract}

Key words: Rheumatoid arthritis. Interstitial lung disease. Citrullination. Anti-fibrotic.

\section{INTRODUCTION}

The prevalence of rheumatoid arthritis (RA) ranges from 0.5 to $1 \%$ in the general population ${ }^{1}$. Although the joint compromise is the most recognized, RA is a systemic disease with many non-articular manifestations. The prevalence of interstitial lung disease (ILD) in patients with RA varies from 10 to $42 \%$ in different

Corresponding author:

*Francisco Paulin

Hospital "María Ferrer"

Doctor Enrique Finochietto, 849

C.P. C1272AAA, Buenos Aires, Argentina

E-mail: francisco_paulin@yahoo.com.ar 
series, depending on the sensitivity of the utilized screening tests 2,3 . The presence of ILD has important prognostic implications since RA patients with ILD have three times the risk of death compared with those without ILD ${ }^{3}$.

Rheumatoid arthritis ILD is associated with almost all the histopathologic patterns of idiopathic interstitial pneumonia. Usual interstitial pneumonia (UIP) is the most prevalent, followed by non-specific interstitial pneumonia (NSIP) and organizing pneumonia ${ }^{4}$. The most practical approach is to classify RA ILD in two different phenotypes: UIP and non-UIP patterns ${ }^{5}$. In this spectrum of histological and high-resolution computed tomography (HRCT) patterns, UIP represents the fibrotic pole, while the non-UIP pattern represents the inflammatory one. Patients with UIP pattern are older, more frequently male and smokers, and have worst prognosis compared to those with the non-UIP pattern ${ }^{6-8}$. The prognosis of patients with RA and UIP pattern mirrors idiopathic pulmonary fibrosis (IPF), with a median survival rate close to three years in both conditions ${ }^{8,9}$. On the other hand, the survival in non-UIP patients is approximately twice as long ${ }^{8}$.

Given the lack of evidence-based recommendations to evaluate and treat patients with RA ILD, the aim of this review is to propose a practical and mechanistic approach to guide the decisions in everyday practice.

\section{MECHANISMS BEHIND THE CONCOMITANCE OF LUNG AND JOINT INVOLVEMENT IN RHEUMATOID ARTHRITIS}

Citrullination is a crucial event for the development of RA, and consists in the conversion of arginine to citrulline, an amino acid that results immunogenic in a group of genetically predisposed individuals ${ }^{10}$. Those individuals show certain human leukocyte antigens (HLA) called "shared epitopes," which give them an increased tendency to bind and present citrullinated peptides ${ }^{11}$. The place where this initial event occurs is a topic of discussion, but most of the evidence points towards mucosal sites (e.g. oral mucosa) ${ }^{12}$. Once antigen presentation occurs, both humoral and cellular immune responses against citrullinated peptides develop in synovial tissue. An increase in the concentrations of interleukins (IL) IL1, IL6, tumor necrosis factor (TNF)
Figure 1. Mechanisms behind the concomitance of lung and joint involvement in rheumatoid arthritis.

Black arrows: sequence of events in the development of nonusual interstitial pneumonia pattern of rheumatoid arthritis interstitial lung disease. White arrows: sequence of events in the development of usual interstitial pneumonia pattern rheumatoid arthritis interstitial lung disease.

ACPA: anti-citrullinated peptide antibody; IL: interleukin; ILD: interstitial lung disease; MMP: matrix metalloproteinase; TGF: tumor growth factor.

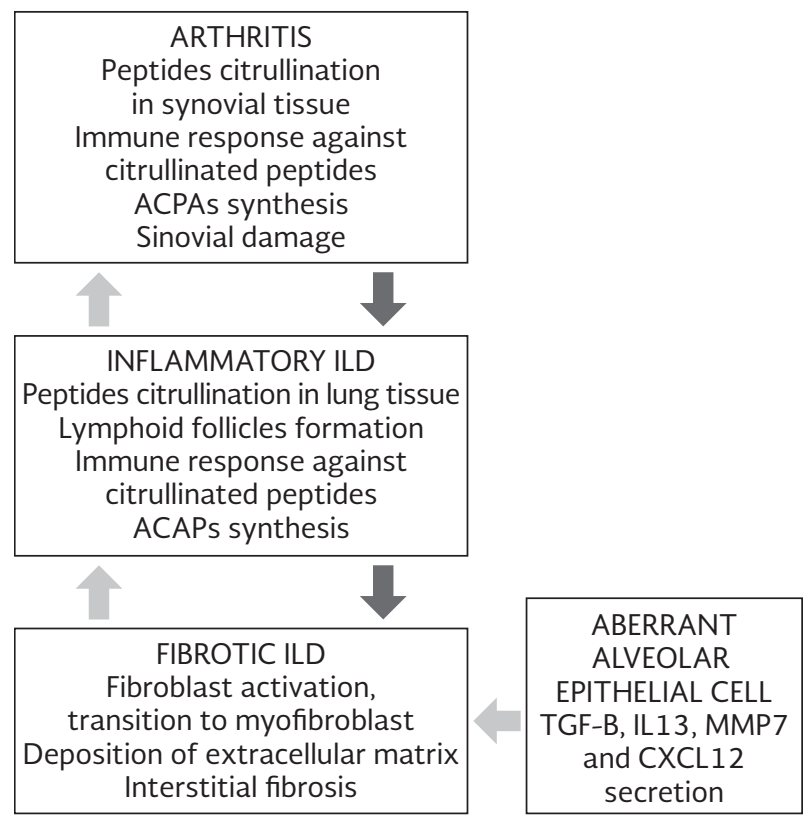

alpha, IL17 and B lymphocyte stimulator, among other humoral mediators, leads to the proliferation of T-cells and differentiation to T-helper cells Thf, Th1 and Th17 profiles. As a consequence, B-lymphocytes produce antibodies (e.g. rheumatoid factor and anticitrullinated protein antibodies [ACPA]), and macrophages and neutrophils release their inflammatory mediators (prostaglandins, proteases, reactive oxygen intermediates, etc.), all of them contributing to tissue injury ${ }^{13}$. Innate immunity receptors, in particular, the Nod-like receptors and inflammasomes, are very important for the initiation and maintenance of the inflammatory cascade ${ }^{14,15}$. As a consequence, both adaptive and innate immune response cells participate in this process, with the last ones playing a key role, specifically in synovial damage.

Some hypotheses exist, trying to explain the concomitance of lung and joint involvement in RA ${ }^{16,17}$ (Fig. 1). Our group proposed that there are two pathways linking ILD and joint compromise in $\mathrm{RA}^{17}$. In the 
Figure 2. Assessment of the phenotype in rheumatoid arthritis associated interstitial lung disease. (1) Clear predominance of ground glass opacities or consolidation.

HRCT: high resolution computed tomography; UIP: usual interstitial pneumonia.

Determine HRCT pattern

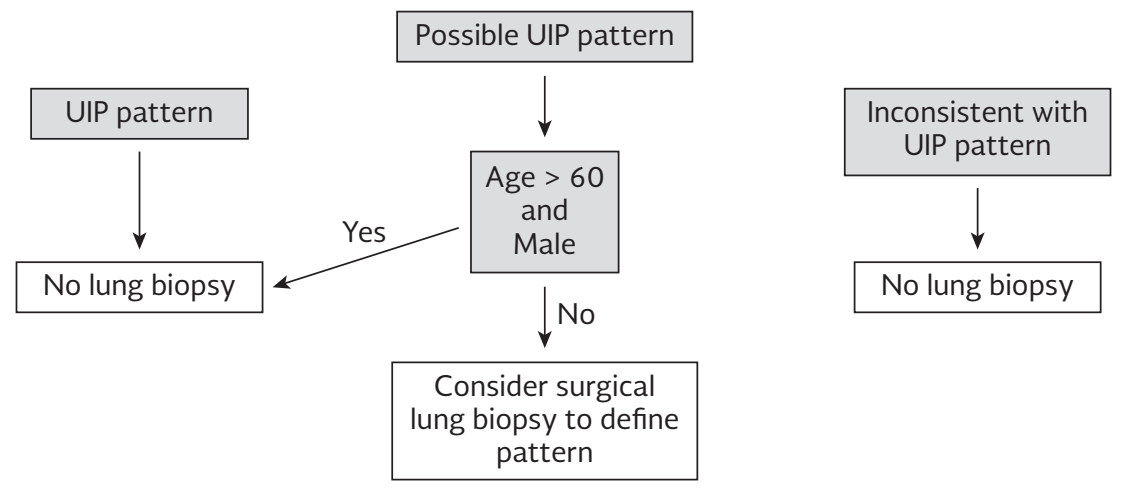

first pathway (called "from the joints to the lungs"), the process begins in the synovial tissue from a patient with established RA. According to this hypothesis, a proportion of RA patients with joint compromise also produce citrullinated peptides in the lung parenchyma. Consequentially, the ACPAs recognize those citrullinated peptides; the immune response moves to the lung and ILD develops ${ }^{17}$. There is an initial inflammatory phase showing $\mathrm{T}$ - and B-cell infiltrates in the lung interstitium, which subsequently leads to a repair phase in which cytokines like IL13, and tumor growth factor (TGF)-B promote the transition of the fibroblast to myofibroblast, the main actor in interstitial fibrosis. This sequence of events probably represents what occurs in the inflammatory pole of RA ILD (nonUIP pattern). In the second pathway (called "from the lungs to the joints"), the process begins in the lung parenchyma of an individual with ILD, in particular, with an IPF-like mechanism ${ }^{17}$. According to the current IPF paradigm, aged alveolar epithelial cells, which are genetically conditioned and subjected to mechanical stress, gain the ability to secrete a variety of substances, including growth factors (TGF-B), chemokines (CXCL12), matrix metalloproteinases (MMP-7), etc. In turn, these substances promote the transition of fibroblast to myofibroblast, which deposits extracellular matrix components and leads to tissue fibrosis ${ }^{17}$. It has been demonstrated that patients with IPF show citrullinated peptides in the lung parenchyma ${ }^{18}$. Therefore, in those with a predisposing genetic background (e.g. shared epitopes), an immune response against citrullinated peptides develops. Finally, ACPAs are synthesized in the lung, and tissues showing citrullinated peptides are targeted (e.g. lung interstitium and synovium). This mechanism probably reflects what occurs in RA UIP pattern, and also explains why the UIP pattern of RA ILD has in some cases inflammatory infiltrates ${ }^{19}$.

It is of note that in the first pathway, the fibrotic process is a consequence of the inflammatory one, but in the second pathway, both fibrotic and inflammatory processes are in parallel. This is an important factor since it may have therapeutic implications. Although the "two pathways hypothesis" in our opinion can explain the whole problem related to RA ILD, it needs to be studied and demonstrated.

\section{APPROACH TO RECOGNIZING DIFFERENT PHENOTYPES AND ASSESSING DISEASE ACTIVITY IN THE LUNGS}

As was shown previously, there are reasons to believe that different phenotypes in RA ILD represent different underlying mechanisms ${ }^{17}$. Therefore, identifying them may prove useful in choosing the most appropriate treatment (an anti-inflammatory agent and/or an anti-fibrotic one). We propose two different strategies to approach the disease phenotype and assess the disease activity (Fig. 2).

\section{Noninvasive strategy}

The HRCT has proven to be an important resource in the evaluation of patients with suspected IPF ${ }^{20}$. As it occurs in IPF, the UIP pattern can be diagnosed with 
very good specificity $(96 \% ; 95 \% \mathrm{Cl}: 81-100)$ and positive predictive value (95\%; $95 \% \mathrm{Cl}: 75-100$ ), but modest sensitivity (45\%; $95 \% \mathrm{Cl}: 30-61$ ) and negative predictive value ( $53 \%$; $95 \% \mathrm{Cl}: 38-68)$ in patients with RA ILD ${ }^{21}$. The correlation between HRCT and pathology fibrosis scores in ILD patients has shown to be acceptable, and the same occurs with the ground glass opacities and inflammatory infiltrate scores ${ }^{22}$. In patients with RA ILD, ground glass scores have shown to correlate with RA disease activity measured with composite indexes, e.g. Clinical Disease Activity Index $(\mathrm{CDAl})^{23}$. This has not proven to be true regarding fibrosis scores in HRCT, which reflects the fact that ILD does not always depend on an inflammatory process. A study that measured the response to diseasemodifying anti-rheumatic drug (DMARD) treatment in patients with RA ILD showed that those patients with lowest fibrosis scores had a better functional response ${ }^{24}$. Collectively, these data suggest that HRCT is useful to define the pattern of lung compromise in patients with ILD, especially in UIP cases, and can contribute to assessing the activity of the disease and response to treatment. Thus, we propose that HRCT should be the first study to be performed on these patients in order to define the ILD phenotype.

Rheumatologists are familiar with the utilization of disease activity scores in daily practice. The most utilized are Disease Activity Score 28 (DAS28) and $\mathrm{CDAl}^{25}$. These are validated instruments with cutoff points that discriminate between remission and mild, moderate, and high disease activity. The presence of moderate-to-high activity, measured by any of these scores in a patient with RA ILD, should raise the suspicion of an inflammatory mechanism behind the lung compromise. In this scenario, interaction between the pulmonologist and the rheumatologist is mandatory to assess the activity level of the disease and consider the best therapy. In patients without clinical evidence of joint tenderness, the utilization of power Doppler or magnetic resonance imaging (MRI) may be useful to detect subclinical synovitis ${ }^{26}$.

The utility of ACPAs to assess the activity of RA ILD has not yet been determined. However, some interesting findings in this direction have been published. The ACPA titers have shown to correlate to the number of lymphoid follicles in the lung biopsy of RA ILD patients in one study ${ }^{27}$. This clearly suggests that the lung contributes to the total pool of circulating ACPAs. It has been demonstrated that patients with RA ILD have higher titers of ACPAs compared with those without ILD, with an OR of 3.83 ( $95 \% \mathrm{Cl}: 1.74-8.43)^{28}$. Moreover, ACPAs are associated with severity and extent of RA ILD in HRCT ${ }^{29}$. As a consequence, the presence of high ACPA titers (defined as an elevation of the titers more than three times the upper limit of normality) in an RA patient with ILD may be evidence of an immune response taking place at the lung ${ }^{30}$.

In patients with ILD, the utilization of biomarkers may represent an interesting strategy for diagnosis or to obtain prognostic information without performing a surgical lung biopsy. Biomarkers were first studied in IPF, where higher levels of MMP-7 and SP-D are associated with reduced survival ${ }^{31,32}$. In RA ILD, a combinatorial signature including MMP-7 and IP-10 (a chemokine related to Th1 lymphocyte trafficking) significantly increased the detection of the disease, while MMP-7 and SP-D have also been shown to enhance our ability to risk-stratify clinically evident and subclinical disease $^{33,34}$. However, the role of these or other biomarkers in clinical practice needs to be clarified.

Summarizing, we consider that HRCT, disease activity scores (DAS28 or CDAl), acute-phase reactants (Creactive protein and erythrocyte sedimentation rate) and ACPA titers and, in selected cases, power Doppler or MRI, can help to recognize an underlying inflammatory phenomenon and direct the treatment strategy.

\section{Invasive strategy}

There is no evidence to guide the decision of when to perform a surgical lung biopsy and, more importantly, what information it can provide in patients with the coexistence of RA and ILD. Given the lack of evidence, we suggest the use of an algorithm that can help select which patients may need a surgical lung biopsy.

The first step is to determine the HRCT pattern. The objective of performing a surgical lung biopsy is to determine the phenotype of ILD. Thus, in patients in whom the phenotype can be assessed with HRCT, the lung biopsy does not seem to have utility. This is the case of patients with a typical UIP pattern, which has shown good correlation with UIP in the biopsy specimen. In this group of patients, the IPF-like mechanism can be assumed, and an appropriate treatment strategy should be implemented. In the same line, 
Figure 3. Rheumatoid arthritis interstitial lung disease treatment according to phenotype.

ILD: interstitial lung disease; RA: rheumatoid arthritis; UIP: usual interstitial pneumonia.

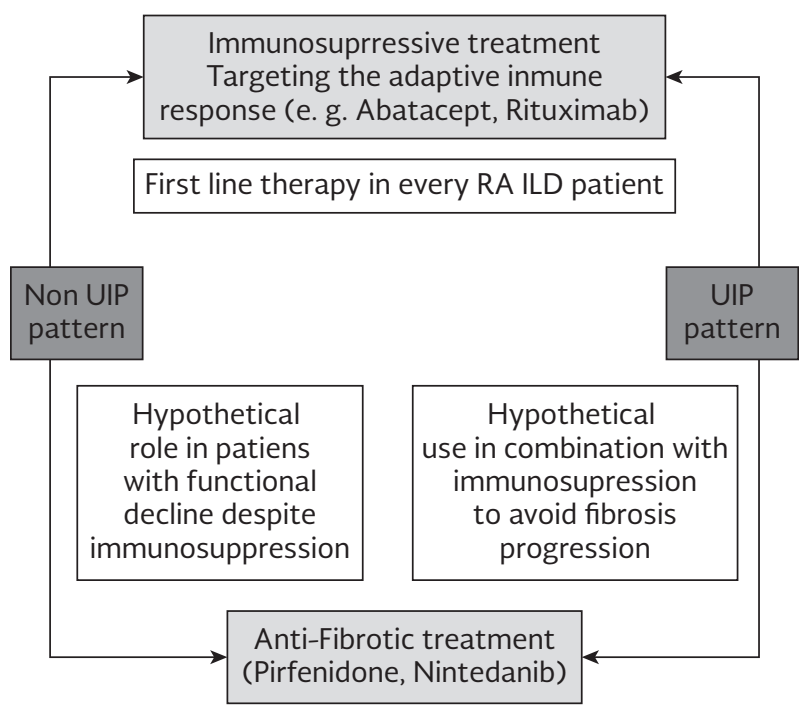

patients with a clearly inflammatory HRCT pattern (with predominating ground glass opacities and/or consolidation) should not be biopsied. An anti-inflammatory treatment should be implemented to avoid the development of secondary fibrosis.

Surgical lung biopsy should be considered in patients with an HRCT that cannot discriminate between these two poles. This is the case of patients with a possible UIP pattern, except for male patients older than 60 years who will probably show a UIP in the biopsy ${ }^{35}$.

\section{THERAPEUTIC APPROACH TO THE INFLAMMATORY PHENOTYPE OF RHEUMATOID ARTHRITIS INTERSTITIAL LUNG DISEASE (FIG. 3)}

\section{Extrapolating from other diseases with a similar behavior in the lung}

The only connective tissue disease-related ILD where randomized controlled trials (RCT) were performed is Scleroderma-related ILD. The most important one is the Scleroderma Lung Study, a multicenter, randomized, cyclophosphamide vs. placebo trial ${ }^{36}$. The same group of researchers has just presented the results of Scleroderma Lung Study II, a comparison between cyclophosphamide and mycophenolate mofetil ${ }^{37}$. Those studies have only shown a subtle benefit for either cyclophosphamide or mycophenolate mofetil. Given the fact that these are the only RCTs in connective tissue disease ILD, and the lack of evidence regarding RA ILD treatment, the possibility of extrapolating them to RA appears attractive, assuming that at least in part they share pathogenic mechanisms. However, we should be very careful when extrapolating treatments from different diseases since they may have diverse results.

In many autoimmune diseases, particularly for the treatment of severe manifestations, the strategy includes an induction phase (more aggressive) and a maintenance phase ${ }^{38}$. As RA ILD has a high mortality, this approach could also be applied in this clinical scenario as well.

\section{Extrapolating from the treatment of other manifestations of rheumatoid arthritis}

The latest American College of Rheumatology recommendations do not consider specifically the situation of patients with RA and ILD, but suggest that extra-articular disease carries a worse prognosis and should receive a more aggressive treatment ${ }^{25}$. Evidence suggests that the mechanisms involved in joint inflammation are different from the ones contributing to lung disease. RA is an autoimmune disease, in which a specific immune response (e.g. against citrullinated peptides) takes place in different organs, directed and controlled by the adaptive immune system. However, the effector arm of the tissue injury varies in the different organs, in particular lungs and synovium. The profile of inflammatory cells contributing to the damage in both sites is quite different. Regardless of the specific histology subtype (UIP, NSIP, etc.), the lung tissue usually shows lymphoid aggregates and even large accumulations of lymphocytes that have the appearance of lymphoid follicles with germinal centers ${ }^{19,27}$. Plasma cells and dendritic cells are also present, but to a lesser amount. On the other hand, macrophages and fibroblast-like synoviocytes are the dominant cellular populations in the synovial tissue of the majority of cases $^{39}$. This diverse cellular profile reveals a different immune response mediating the tissue damage in both sites, with an innate immune profile acting in the synovium and an adaptive immune response contributing to the lung injury. 
The DMARDs play an important role in the treatment of RA. In fact, in the majority of cases they represent the first treatment option ${ }^{39}$. However, an increasing number of reports have appeared regarding the lung toxicity of this group of drugs ${ }^{40}$. Methotrexate is the most utilized and paradigmatic drug from this group, and the occurrence of lung disease in patients receiving this drug has also been reported. This fact has generated confusion regarding the use of this agent in patients with RA ILD. The reported lung toxicity with methotrexate is of two different types: an acute hypersensitivity pneumonitis and a chronic ILD ${ }^{40}$. Nevertheless, some interesting aspects should be considered. Methotrexate is widely used to treat psoriasis, a disease that does not affect the lungs. In patients with psoriasis receiving methotrexate, the reported lung toxicity is notably much less prevalent than in RA patients ${ }^{41-45}$. Furthermore, a study showed that the prevalence of chronic lung disease (screened with HRCT and pulmonary function tests) in RA patients receiving methotrexate does not seem to be greater than in patients receiving other treatments ${ }^{46}$. Furthermore, methotrexate has proven to improve lung function in a small observational study of RA ILD ${ }^{24}$. Taken together, these results suggest that what was previously considered chronic lung toxicity due to methotrexate, could in fact be related to RA itself. Based on this information, we consider that methotrexate should not necessarily be suspended in patients with RA who develop chronic ILD. However, another medication that targets lung disease should be added. On the other hand, methotrexate should be discontinued in patients who develop acute ILD, especially if there is a chronological relationship between hypersensitivity pneumonitis pattern and the initiation of methotrexate. Finally, the decision of starting methotrexate in a patient who already has ILD should be carefully considered. The development of acute lung injury secondary to drug toxicity in a patient with a previously abnormal lung function test may be life threatening. The level of lung functional impairment may help guide this difficult decision.

The TNF inhibitor group was the first group of biologic agents that appeared as an option to treat refractory cases of RA. However, the development or worsening of preexisting ILD has been increasingly reported 47,48 . This represents a paradox since this group of drugs has shown to be effective for the treatment of joint compromise ${ }^{25}$. A possible explanation is that anti-TNF agents target a cytokine that plays a key role in the innate immune response taking place in the joint tissue, but does not block the adaptive immune response that mediates the lung disease. As a consequence, we consider that anti-TNF agents should be avoided in patients with previously diagnosed ILD. We also recommend the screening of ILD with HRCT in patients before receiving any anti-TNF agents to avoid the worsening of an undiagnosed ILD.

Rituximab is a monoclonal antibody that targets Blymphocytes by binding to CD20. Its efficacy for the treatment of RA has been demonstrated in various $\mathrm{RCTs}^{49}$. Given the fact that B-lymphocyte infiltrates are prominent in lung biopsies from RA ILD patients, using rituximab to treat RA ILD appears to be an attractive option. Although RCTs demonstrating rituximab's efficacy in RA ILD are lacking, an increasing number of case series showing patient improvements are being published ${ }^{50}$. We consider that rituximab could represent an interesting alternative in the treatment of RA ILD.

Abatacept is a fusion protein (CTLA4-lg) that binds to co-stimulatory molecules CD80 and CD86 in the antigen-presenting cell, blocking its interaction with CD28 in the T lymphocyte surface. Through this mechanism, it prevents antigen presentation and the interaction between $\mathrm{B}$ - and $\mathrm{T}$-cells ${ }^{51}$. Abatacept has largely proven its efficacy for the treatment of joint involvement in RA ${ }^{52}$. Moreover, abatacept has been proposed as an option for the treatment of RA ILD based in several case series ${ }^{53}$. We consider that abatacept is a very promising option in the treatment of RA ILD patients.

\section{THERAPEUTIC APPROACH OF THE FIBROTIC/USUAL INTERSTITIAL PNEUMONIA PHENOTYPE OF RHEUMATOID ARTHRITIS INTERSTITIAL LUNG DISEASE: PUTATIVE ROLE FOR ANTIFIBROTIC AGENTS}

A change in the paradigm of IPF pathogenesis has occurred in the last few years ${ }^{54}$. As a consequence, new therapeutic options have emerged, targeting the fibrotic process itself.

Pirfenidone is a small and simple molecule with multiple effects ${ }^{55}$. The most remarkable is that it reduces 
the levels of TGF-beta, platelet-derived growth factor (PDGF) and fibroblast growth factor (FGF), growth factors that activate the fibroblast and enhance its capacity of depositing extracellular matrix. Through these mechanisms, it targets the fibrotic process and slows the progression of IPF. Pirfenidone has proven to reduce the forced vital capacity decline in IPF patients in four RCTs ${ }^{16,17,56-58}$. Interestingly, pirfenidone reduces the levels of TNF-alpha and IL-6, both cytokines related to the activation of the macrophage and with proven roles in RA pathogenesis ${ }^{55}$.

Nintedanib is a tyrosine kinase inhibitor. It blocks the receptor of three growth factors that are of crucial importance for the development of lung fibrosis: PDGF, vascular endothelial growth factor (VEGF) and FGF59. Through this mechanism, it interferes with fibroblast proliferation and differentiation, together with its capacity of producing the extracellular matrix. It has proven to slow forced vital capacity decline in patients with IPF in two RCTs ${ }^{60,61}$. Given the similarities between IPF and RA UIP, it makes sense to propose that these two drugs may have a role in the treatment of RA ILD patients with this specific phenotype. Based on our hypothesis, targeting the inflammatory process with immunosuppressants alone will not prevent the progression of the fibrotic process and functional decline. The association of both types of agents (immunosuppressant and anti-fibrotic) can potentially be the future of treatment in this spectrum of the disease.

The utilization of anti-fibrotic agents in patients with a fibrotic NSIP that shows functional deterioration in spite of anti-inflammatory treatment could make sense. However, since in this group of patients fibrosis appears as a secondary process, the first drug to introduce should be an immunosuppressant.

At present, the utilization of anti-fibrotic drugs in patients with RA ILD is purely speculative since there is no evidence to support it. This situation warrants the need for trials evaluating the effectiveness of antifibrotic agents in this group of patients. On the other hand, given the inflammatory nature of RA and the evidence available about its efficacy for the treatment of joint involvement, the utilization of agents that target the immune system is the only strategy we can support.

\section{CONCLUSIONS}

Evidence that defines which is the most adequate approach for the evaluation and treatment of RA ILD patients is lacking. In this scenario, extrapolating evidence from similar clinical situations can be useful. Furthermore, revising the pathogenic mechanisms can help in understanding the clinical behavior of the disease and choosing the most appropriate treatment available to target those mechanisms. Polarizing this heterogeneous group of patients in UIP and non-UIP patterns may contribute to simplifying this therapeutic dilemma. We propose a practical approach to organize the available information and clarify the more conflicting aspects of RA ILD.

\section{REFERENCES}

1. Gabriel SE. The epidemiology of rheumatoid arthritis. Rheum Dis Clin North Am. 2001;27:269-81.

2. Zou Y-Q, Li Y-S, Ding X-N, Ying Z-H. The clinical significance of HRCT in evaluation of patients with rheumatoid arthritis-associated interstitial lung disease: a report from China. Rheumatol Int. 2012;32:669-73

3. Olson AL, Swigris J], Sprunger DB, et al. Rheumatoid arthritis interstitial lung disease associated mortality. Am J Respir Crit Care Med. 2011;183:372-8

4. Lee H, Kim DS, Yoo B. Histopathologic pattern and clinical features of rheumatoid arthritis-associated interstitial lung disease. Chest. 2005;127:2019-27.

5. Assayag D, Lee JS, King TE. Rheumatoid arthritis associated interstitial lung disease: a review. Medicina (B Aires). 2014;74: 158-65

6. Assayag D, Elicker BM, Urbania $\mathrm{TH}$, et al. Rheumatoid arthritis - associated interstitial lung disease : Radiologic identification of usual interstitial pneumonia pattern. Radiology. 2014;270: 583-8.

7. Kim EJ, Collard HR, King TE. Rheumatoid arthritis-associated interstitial lung disease: the relevance of histopathologic and radiographic pattern. Chest. 2009:136:1397-405.

8. Kim EJ, Elicker BM, Maldonado F, et al. Usual interstitial pneumonia in rheumatoid arthritis-associated interstitial lung disease. Eur Respir J. 2010;35:1322-8.

9. Ragu G. An Official ATS/ERS/JRS/ALAT Statement: Idiopathic Pulmonary Fibrosis: Evidence-based Guidelines for Diagnosis and Management. Am J Respir Crit Care Med. 2011;183:788-824.

10. Scally SW, Petersen J, Law SC, et al. A molecular basis for the association of the HLA-DRB1 locus, citrullination, and rheumatoid arthritis. J Exp Med. 2013;210:2569-82.

11. Klareskog L, Padyukov L, Lorentzen J, Alfredsson L. Mechanisms of disease: Genetic susceptibility and environmental triggers in the development of rheumatoid arthritis. Nat Clin Pract Rheumatol. 2006;2:425-33

12. Demoruelle MK, Deane KD, Holers VM. When and where does inflammation begin in rheumatoid arthritis? Curr Opin Rheumatol. 2014;26:64-71.

13. Mclnnes IB, Schett G. The pathogenesis of rheumatoid arthritis. N Engl J Med. 2011 8;365:2205-19.

14. Yang $C A$, Chiang BL. Inflammasomes and human autoimmunity: A comprehensive review. J Autoimmun. 2015;61:1-8.

15. Choulaki C, Papadaki G, Repa A, et al. Enhanced activity of NLRP3 inflammasome in peripheral blood cells of patients with active rheumatoid arthritis. Arthritis Res Ther. 2015;17:257.

16. Chatzidionisyou A, Catrina Al. The lung in rheumatoid arthritis, cause or consequence? Curr Opin Rheumatol. 2016;28:76-82.

17. Paulin F, Doyle TJ, Fletcher EA, Ascherman DP, Rosas IO. Rheumatoid arthritis-associated interstitial lung disease and idiopathic pulmonary fibrosis: Shared mechanistic and phenotypic 
traits suggest overlapping disease mechanisms. Rev Investig Clin. 2015;67:280-6

18. Bongartz T, Cantaert T, Atkins SR, et al. Citrullination in extraarticular manifestations of rheumatoid arthritis. Rheumatology (Oxford). 2007;46:70-5

19. Wuyts WA, Cavazza A, Rossi G, Bonella F, Sverzellati N, Spagnolo P. Differential diagnosis of usual interstitial pneumonia: when is it truly idiopathic? Eur Respir Rev. 2014;23:308-19.

20. Raghu G, Collard HR, Egan JJ, et al. An official ATS/ERS/JRS/ ALAT statement: idiopathic pulmonary fibrosis: evidence-based guidelines for diagnosis and management. Am J Respir Crit Care Med. 2011;183:788-824.

21. Assayag D, Elicker BM, Urbania TH, et al. Rheumatoid arthritisassociated interstitial lung disease: radiologic identification of usual interstitial pneumonia pattern. Radiology. 2014;270:583-8.

22. Kazerooni EA, Martinez FJ, Flint A, et al. Thin-section CT obtained at $10-\mathrm{mm}$ increments versus limited three-level thinsection CT for idiopathic pulmonary fibrosis: correlation with pathologic scoring. AJR Am J Roentgenol. 1997;169:977-83.

23. Pérez-Dórame R, Mejía M, Mateos-Toledo H, Rojas-Serrano J. Rheumatoid arthritis-associated interstitial lung disease: lung inflammation evaluated with high resolution computed tomography scan is correlated to rheumatoid arthritis disease activity. Reumatol Clin. 2015;11:12-16.

24. Rojas-Serrano J, González-Velásquez E, Mejía M, Sánchez-Rodríguez A, Carrillo G. Interstitial lung disease related to rheumatoid arthritis: evolution after treatment. Reumatol Clin. 2012;8:68-71.

25. Singh JA, Furst DE, Bharat A et al 2012 update of the 2008 American College of Rheumatology recommendations for the use of disease-modifying antirheumatic drugs and biologic agents in the treatment of rheumatoid arthritis. Arthritis Care Res (Hoboken). 2012;64:625-39.

26. Mathew AJ, Danda D, Conaghan PG. MRI and ultrasound in rheumatoid arthritis. Curr Opin Rheumatol. 2016;28:323-9.

27. Rangel-Moreno J, Hartson L, Navarro C, Gaxiola M, Selman M Randall TD. Inducible bronchus-associated lymphoid tissue (iBALT) in patients with pulmonary complications of rheumatoid arthritis. J Clin Invest. 2006;116:3183-94.

28. Young A. Rheumatoid arthritis-related interstitial lung disease: associations, prognostic factors and physiological and radiological characteristics--a large multicentre UK study. Rheumatology (Oxford). 2014:53:1676-82.

29. Rocha-Muñoz AD, Ponce-Guarneros M, Gamez-Nava Jl, et al. Anti-cyclic citrullinated peptide antibodies and severity of interstitial lung disease in women with rheumatoid arthritis. J Immunol Res. 2015:2015:1-10.

30. Aletaha D, Neogi T, Silman AJ, et al. 2010 Rheumatoid arthritis classification criteria: an American College of Rheumatology/ European League Against Rheumatism collaborative initiative. Arthritis Rheum. 2010;62:2569-81.

31. Richards TJ, Kaminski N, Baribaud F, et al. Peripheral blood proteins predict mortality in idiopathic pulmonary fibrosis. Am J Respir Crit Care Med. 2012;185:67-76.

32. Takahashi H, Fujishima T, Koba H, et al. Serum surfactant proteins $A$ and $D$ as prognostic factors in idiopathic pulmonary fibrosis and their relationship to disease extent. Am J Respir Crit Care Med. 2000;162:1109-14

33. Doyle TJ, Patel AS, Hatabu H, et al. Detection of rheumatoid arthritis-interstitial lung disease is enhanced by serum biomarkers. Am J Respir Crit Care Med. 2015;191:1403-12.

34. Chen J, Doyle TJ, Liu Y, et al. Biomarkers of rheumatoid arthritisassociated interstitial lung disease. Arthritis Rheumatol. 2015; 67:28-38.

35. Bois RM. An earlier and more confident diagnosis of idiopathic pulmonary fibrosis. Eur Respir Rev. 2012;21:141-6

36. Tashkin DP, Elashoff R, Clements PJ, et al. Effects of 1-year treatment with cyclophosphamide on outcomes at 2 years in scleroderma lung disease. Am J Respir Crit Care Med. 2007;176: 1026-34.

37. Tashkin DP, Roth MD, Clements PJ, et al. Mycophenolate mofetil versus oral cyclophosphamide in scleroderma-related interstitial lung disease (SLS II): a randomised controlled, double-blind, parallel group trial. Lancet Respir Med. 2016;4:708-19.

38. Hahn BH, McMahon MA, Wilkinson A, et al. American College of Rheumatology guidelines for screening, treatment, and management of lupus nephritis. Arthritis Care Res. 2012;64:797-808.
39. Tak PP, Bresnihan B. The pathogenesis and prevention of joint damage in rheumatoid arthritis: advances from synovial biopsy and tissue analysis. Arthritis Rheum. 2000;43:2619-33.

40. Roubille C, Haraoui B. Interstitial lung diseases induced or exacerbated by DMARDS and biologic agents in rheumatoid arthritis: A systematic literature review. Semin Arthritis Rheum. 2014; 43:613-26

41. Belzunegui J, Intxausti JJ, De Dios JR, et al. Absence of pulmonary fibrosis in patients with psoriatic arthritis treated with weekly low-dose methotrexate. Clin Exp Rheumatol. 2001;19: 727-30.

42. Conway R, Low C, Coughlan RJ, O'Donnell MJ, Carey JJ. Methotrexate use and risk of lung disease in psoriasis, psoriatic arthritis, and inflammatory bowel disease: systematic literature review and meta-analysis of randomised controlled trials. BMJ. 2015;350:h1269.

43. Haustein UF, Rytter M. Methotrexate in psoriasis: 26 years experience with low-dose long-term treatment. J Eur Acad Dermatol Venereol. 2000;14:382-8.

44. McKenna KE, Burrows D. Pulmonary toxicity in a patient with psoriasis receiving methotrexate therapy. Clin Exp Dermatol. 2000;25:24-7

45. Salaffi F, Manganelli P, Carotti M, Subiaco S, Lamanna G, Cervini C. Methotrexate-induced pneumonitis in patients with rheumatoid arthritis and psoriatic arthritis: report of five cases and review of the literature. Clin Rheumatol. 1997;16:296-304

46. Dawson JK, Graham DR, Desmond J, Fewins HE, Lynch MP. Investigation of the chronic pulmonary effects of low-dose ora methotrexate in patients with rheumatoid arthritis: a prospective study incorporating HRCT scanning and pulmonary function tests. Rheumatology (Oxford). 2002;41:262-7.

47. Koo BS, Hong S, Kim YJ, Kim Y-G, Lee C-K, Yoo B. Mortality in patients with rheumatoid arthritis-associated interstitial lung disease treated with an anti-tumor necrosis factor agent. Korean J Intern Med. 2015:30:104-9.

48. Nakashita T, Ando K, Kaneko N, Takahashi K, Motojima S. Potential risk of TNF inhibitors on the progression of interstitial lung disease in patients with rheumatoid arthritis. BMJ Open. 2014.4.e005615.

49. Mok CC. Rituximab for the treatment of rheumatoid arthritis: an update. Drug Des Devel Ther. 2014;8:87-100.

50. Chartrand S, Swigris JJ, Peykova L, Fischer A. Rituximab for the treatment of connective tissue disease-associated interstitial lung disease. Sarcoidosis Vasc Diffuse Lung Dis. 2016;32:296-304.

51. Herrero-Beaumont G, Martínez Calatrava MJ CS. Abatacept mechanism of action: concordance with its clinical profile. Reum Clin. 2012;8:78-83.

52. Keystone E, Alkhalaf A, Makkawy M. Subcutaneous abatacept in rheumatoid arthritis: current update. Expert Opin Biol Ther. 2015;15:1221-30

53. Mera-Varela A, Pérez-Pampín E. Abatacept therapy in rheumatoid arthritis with interstitial lung disease. J Clin Rheumatol. 2014:20:445-6

54. Selman M, Pardo A. Revealing the pathogenic and aging-related mechanisms of the enigmatic idiopathic pulmonary fibrosis: An integral model. Am J Respir Crit Care Med. 2014:189:1161-72.

55. Schaefer CJ, Ruhrmund DW, Pan L, Seiwert SD, Kossen K. Antifibrotic activities of pirfenidone in animal models. Eur Respir Rev. 2011;20:85-97.

56. Taniguchi $\mathrm{H}$, Ebina $\mathrm{M}$, Kondoh $\mathrm{Y}$, et al. Pirfenidone in idiopathic pulmonary fibrosis. Eur Respir J. 2010;35:821-9.

57. King TE, Bradford WZ, Castro-Bernardini S, et al. A Phase 3 trial of pirfenidone in patients with idiopathic pulmonary fibrosis. $\mathrm{N}$ Engl J Med. 2014;22370:2083-92

58. Noble PW, Albera C, Bradford WZ, et al. Pirfenidone in patients with idiopathic pulmonary fibrosis (CAPACITY): two randomized controlled trials Lancet. 2011:377:1760-8.

59. Wollin L, Wex E, Pautsch A, et al. Mode of action of nintedanib in the treatment of idiopathic pulmonary fibrosis. Eur Respir J. 2015:45:1434-45.

60. Richeldi Luca, Costabel Ulrich, Selman Moises, et al. Efficacy of a tyrosine kinase inhibitor in Idiopathic pulmonary fibrosis. $\mathrm{N}$ Engl J Med. 2011:365:1079-87.

61. Richeldi L, Du Bois RM, Raghu G, et al. Efficacy and safety of nintedanib in idiopathic pulmonary fibrosis. N Engl J Med. 2014; 370:2071-82. 